\title{
Variational Principle for Linear Relaxation Relations
}

\author{
S. Grossmann \\ Fachbereich Physik, Marburg \\ Z. Naturforsch. 34a, 1275-1278 (1979); received August 25, 1979
}

A variational principle for linear relaxation phenomena is considered. It connects relaxation with anti-relaxation. The latter one is governed by the transposed transport matrix and, in addition, by the diffusion matrix, which drops if Onsager-symmetry holds. This generalizes an earlier result by $\mathrm{L}$. Waldmann.

1. Recently Waldmann pointed out that linear transport relaxation equations can be derived from a variational principle, if certain time reversal symmetries hold [1]. He postulated an action with a quadratic Lagrangian, which implies by its extremum the relaxation equations as its EulerLagrange equations.

In the following i) the relation of Waldmann's action to the Onsager-Machlup [2] theory is pointed out. It determines the probability of the accompanying stochastic process $\propto \exp$ (-action). ii) It is not necessary to refer to time reversal properties; in fact, there are physical systems which do not show these but nevertheless allow for a (slightly generalized) action. An example is the Brusselator [3], a chemically reacting system capable of a limit cycle, i.e. of broken time translational invariance. iii) The anti-relaxation solution is not a useless artifact but is a physically necessary element of the most probable path as well as of the stationary state distribution.

These conclusions can be drawn by deducing the variational principle from the stochastic aspects of irreversible linear relaxation processes, in particular from the underlying Langevin equation, using the methods of path integrals, see e.g. [4].

2 . Let $a(t)=\left\{a_{i}(t)\right\}$ be the set of macroscopic variables of interest. The mean equations of motion are supposed to be $\left\langle\dot{a}_{i}\right\rangle=-\lambda_{i j}\left\langle a_{j}\right\rangle$ with constant relaxation matrix $\lambda$. In general $\lambda$ is not symmetric. The influence of the microscopic degrees of freedom is described by fluctuating forces $\xi(t)=\left\{\xi_{i}(t)\right\}$ with zero mean and Gaussian probability distribution

$$
P\left(\left\{\xi_{i}(t)\right\}\right) \propto \exp \left(-\frac{1}{2} \int_{t_{1}}^{t_{2}} \xi_{i}(t) \xi_{i}(t) \mathrm{d} t\right) .
$$

Reprint requests to Prof. Dr. S. Grossmann, Fachbereich Physik, Renthof 6, D-3550 Marburg.
Thus $\left\langle\xi_{i}(t) \xi_{j}\left(t^{\prime}\right)\right\rangle=\delta_{i j} \delta\left(t-t^{\prime}\right)$. The Langevin equation for the linear relaxation process reads

$$
\dot{a}_{i}=-\lambda_{i j} a_{j}+g_{i j} \xi_{j}(t) .
$$

The constant coupling matrix $g$ is supposed to be non-singular.

The statements (i) to (iii) can be deduced from Eqs. (1) and (2), defining the process.

Equation (2) implies

$$
\xi_{i}(t)=g_{i j}^{-1}\left(\dot{a}_{j}+\lambda_{j k} a_{k}\right) .
$$

The probability distribution of the macroscopic variables is

$$
\varrho(\{a(t)\})=P(\{\xi(t)\}) \frac{\partial(\xi)}{\partial(a)} .
$$

The Jacobian is constant, since Eq. (2) is linear, and can by incorporated into the normalization. Thus

$$
\begin{aligned}
\varrho\left(\left\{a_{i}(t)\right\}\right) \propto \exp \left(-\frac{1}{2} \int_{t_{1}}^{t_{2}}\left(\dot{a}_{j}+\lambda_{j m} a_{m}\right)\right. \\
\left.\cdot D_{j k}^{-1}\left(a_{k}+\lambda_{k n} a_{n}\right) \mathrm{d} t\right)
\end{aligned}
$$

is the probability that a path $a_{i}(t)$ from $t_{1}$ to $t_{2}$ is realized. The width of the distribution is determined by the diffusion matrix $D$, symmetric by definition,

$$
D_{j k}=g_{j l} g_{k l}, \quad D=g \tilde{g}=\tilde{D} .
$$

The exponent shall be called "action"

$$
S=\frac{1}{2} \int_{t_{1}}^{t_{2}}(\dot{a}+\lambda a)^{\sim} D^{-1}(\dot{a}+\lambda a) \mathrm{d} t
$$

General stochastic Gaussian processes (2) have already been considered by Fox and Uhlenbeck [5]. They generalize the Onsager-Machlup theory by using even together with odd variables; but they confine themselves to linear deviations from thermal equilibrium, so entropy and Onsager symmetry of the kinetic matrix is at hand.

0340-4811 / $79 / 1100-1275 \$ 01.00 / 0$. - Please order a reprint rather than making your own copy. 
These restrictions are not necessary. One can also treat open systems far from equilibrium by (1), (2) or its explicit solution (3). At first a variational principle is considered, and its connection with Waldmann's principle is studied. An example which violates Onsager symmetry is given. Nevertheless a fluctuation-dissipation (or an Einstein-) relation exists connecting relaxation, stationary correlations, and diffusion.

3. The most probable path between two times $t_{1}, t_{2}$ is determined by the minimum of the exponent,

$$
\delta S:=\delta\left[\frac{1}{2} \int_{t \mathrm{t}}^{t_{2}}(\dot{\tilde{a}}+\tilde{a} \tilde{\hat{\lambda}}) D^{-1}(\dot{a}+\lambda a) \mathrm{d} t\right]=0 .
$$

This variational principle is a slight generalization of Waldmann's postulate [1]

$$
\delta\left[\frac{1}{2} \int_{t_{1}}^{t_{2}}(\dot{\tilde{a}} \theta \dot{a}+\tilde{a} \tilde{\lambda} \theta \lambda a) \mathrm{d} t\right]=0 .
$$

Instead of the time reversal operator $\theta$ with $\tilde{\theta}=\theta$ and $\theta^{2}=1$ the action (4) displays the diffusion matrix $D^{-1}$. This is symmetric, too, although in general $D^{2} \neq 1$; a constant factor in (4) is arbitrary, however. In addition, $D$ is positive definite, whereas $\theta$ has eigenvalues \pm 1 .

Waldmann considers relaxation equations derived from Boltzmann's transport theory. An essential property of the relaxation matrix then is $[6,7]$

$$
\theta \lambda=\tilde{\lambda} \theta \text {. }
$$

This is Onsager's symmetry $\theta L \theta=\tilde{L}$ for the kinetic matrix $L=\lambda \Gamma$ if the static correlation matrix $\Gamma_{i j}=\left\langle a_{i} a_{j}\right\rangle$ is one (take properly chosen variables).

In general, the analogous property of the variational principle (4),

$$
\lambda D=D \tilde{\lambda},
$$

is not satisfied. Nevertheless, the minimum principle (4) holds. The Euler-Lagrange equations are of second order. They factorize into the (non stochastic) relaxation equation and the anti-relaxation equation. But, different from the particular Boltzmann transport equations with Onsager-symmetry $\left(5^{\prime}\right)$ of the relaxation matrix, the anti-relaxation solution in general contains $D$ explicitly.
4. Consider first the case that $\lambda D$ is a symmetric matrix, i.e. property (6) is fulfilled. Then

$$
\begin{array}{r}
\delta \int_{t_{1}}^{t_{2}}\left(\dot{\tilde{a}} D^{-1} \lambda a+\tilde{a} \tilde{\lambda} D^{-1} \dot{a}\right) \mathrm{d} t \\
=\delta\left[\tilde{a} D^{-1} \lambda a\right]_{t_{1}}^{t_{2}} \equiv 0
\end{array}
$$

for the most probable path. Thus (4) reduces to

$$
\delta \frac{1}{2} \int_{t_{1}}^{t_{2}}\left(\dot{\tilde{a}} D^{-1} \dot{a}+\tilde{a} \tilde{\lambda} D^{-1} \lambda a\right) \mathrm{d} t=0,
$$

which corresponds to Waldmann's equation, Equation (5). The Euler-Lagrange equations of (7) do not contain $D^{-1}$. Multiply by $D$,

$$
\left(-\partial_{t}+\lambda\right)\left(\partial_{t}+\lambda\right) a=0 .
$$

The general solution is a superposition of the relaxation solution $\propto e^{-\lambda t}$ and the anti-relaxation solution $\propto e^{+\lambda t}$,

$$
a(t)=e^{-\lambda t} c_{1}+e^{+\lambda t} c_{2} .
$$

There are two constant vectors $c_{1}, c_{2}$, since (8) is of second order. The value of the action $S$ at the minimum dependends on $D$, of course. It is positive, since $D$ is positive definite.

5. An example of a system which violates the symmetry $(6)$ is the chemical reaction system

$$
\begin{aligned}
& A \rightarrow X, \quad 2 X+Y \rightarrow 3 X, \\
& B+X \rightarrow Y+D, \quad X \rightarrow E,
\end{aligned}
$$

called the Brusselator [3]. There is a net flow $A+B \rightarrow D+E$. (10) is an open system, which shows a transition to a limit cycle, if the (properly normalized) concentration $\beta$ of the reservior $B$ exceeds the critical value $\beta_{\mathrm{c}}=1+\alpha^{2} \quad(\alpha$ is the $A$ concentration). In quasi-linear relaxation approximation the master equation appropriate to describe the (spatially homogeneous) reaction (10) reduces to Eq. (2) with

$\lambda=\left(\begin{array}{cr}1-\beta & -\alpha^{2} \\ \beta & \alpha^{2}\end{array}\right)$, and $D=\alpha\left(\begin{array}{cr}1+\beta & -\beta \\ -\beta & \beta\end{array}\right)$,

see [8]. The variables $a_{i}$ are the concentrations of the intermediates $X, Y$ (both even under time reversal). One easily verifies that $\lambda D$ is not symmetric,

$$
\lambda D=\alpha\left(\begin{array}{cc}
1-\beta^{2}+\beta \alpha^{2} & \beta\left(\beta-\beta_{\mathrm{c}}\right) \\
\beta\left(\beta-\beta_{\mathrm{c}}+2\right) & -\beta+\beta \alpha^{2}
\end{array}\right) .
$$


6. The general Euler-Lagrange equations of the action $S$ as defined in Eq. (4) are

$$
\left(-\partial_{t}+\tilde{\lambda}\right) D^{-1}\left(\partial_{t}+\lambda\right) a=0 .
$$

Equation (2) without noise satisfies (12), again. But the anti-relaxation solution is governed now i) by $\exp (\tilde{\lambda} t)$ instead of $\exp (\lambda t)$ and, in addition, ii) by a (renormalized) diffusion matrix $\hat{D}(t)$. Put $b=$ $D^{-1}(\dot{a}+\lambda a)$. It satisfies the anti-equation

$$
\dot{b}=\tilde{\lambda} b \text {. }
$$

$b(t)=e^{\tilde{\lambda} t} b_{1}=D^{-1}(\dot{a}+\lambda a)$ implies as the general two parameter solution of Eq. (12):

$$
\begin{aligned}
a(t) & =\int_{t_{1}}^{t} \mathrm{~d} t^{\prime} e^{\lambda\left(t^{\prime}-t\right)} D e^{\tilde{\lambda} t^{\prime}} b_{1}+e^{-\lambda t} b_{2} \\
& =\hat{D}(t) e^{\tilde{\lambda} t} b_{1}+e^{-\lambda t} b_{3},
\end{aligned}
$$

with the time-dependent, symmetric matrix

$$
\hat{D}(t)=\int_{0}^{t} e^{-\lambda \tau} D e^{-\tilde{\lambda} \tau} \mathrm{d} \tau .
$$

It is $\hat{D}(0)=0$. The limit $\hat{D}(t \rightarrow \infty)=: \Gamma$ will turn out to be the static correlation matrix $\Gamma_{i j}=\left\langle a_{i} a_{j}\right\rangle$.

$$
\Gamma=\int_{0}^{\infty} e^{-\lambda \tau} D e^{-\tilde{\lambda} \tau} \mathrm{d} \tau .
$$

(It is assumed that $\lambda$ and $\tilde{\lambda}$ have eigenvalues with positive real part and the limit $t \rightarrow \infty$ exists.)

$\Gamma$ satisfies the relation

$$
\lambda \Gamma+\Gamma \tilde{\lambda}=D .
$$

Use $\lambda \hat{D}(t)+\hat{D}(t) \tilde{\lambda}=D-e^{-\lambda t} D e^{-\tilde{\lambda} t}$.

7. $L=\lambda \Gamma$ is the matrix of the kinetic coefficients which in irreversible processes near equilibrium obays the Onsager symmetry $[7,9]$

$$
\tilde{L}=\theta L \theta
$$

due to detailed balancing or microscopic time reversal symmetry. $L$ connects the fluxes and forces, $\dot{a}=-L X$.

Onsager and Machlup in [2] confine their paper to systems with a symmetric kinetic matrix, $L=\tilde{L}$, which implies $D=2 \lambda \Gamma=2 \Gamma \tilde{\lambda}$. Consequently $\lambda D=2 \lambda \Gamma \tilde{\lambda}$ is symmetric, i.e. relation $(6)$ is fulfilled. In general, for an arbitrary relaxation matrix $\lambda$, the kinetic matrix $L$ is not symmetric (although $\Gamma$ as well as $D$ in (17) are so, by definition). This occurs in particular in open, stationary non- equilibrium states. For chemically reacting systems this has been discussed for instance by Jähnig and Richter [10].

Consider as an example the Brusselator again. One can solve the $2 \times 2$ Eqs. (17) for $\Gamma$, using (11).

$$
\Gamma=\frac{\alpha}{\beta_{\mathrm{c}}-\beta}\left(\begin{array}{cc}
\frac{1}{2}\left(\beta+\beta_{\mathrm{c}}\right) & -\beta \\
-\beta & \frac{\beta}{2 \alpha^{2}}\left(\beta+\beta_{\mathrm{c}}\right)
\end{array}\right), \quad \beta<\beta_{\mathrm{c}} .
$$

The kinetic matrix $L$ is not symmetric:

$$
L=\lambda \Gamma=\frac{\alpha}{2}\left(\begin{array}{cc}
1+\beta & -\beta\left(1+\frac{2}{\beta_{\mathrm{c}}-\beta}\right) \\
-\beta\left(1-\frac{2}{\beta_{\mathrm{c}}-\beta}\right) & \beta
\end{array}\right) .
$$

Note that the general solution (14) reduces to the special one, Eq. (9), if one could use the symmetry (6), with $c_{2}=\frac{1}{2} \lambda^{-1} D b_{1}$ and $c_{1}=-\frac{1}{2} \lambda^{-1} D b_{1}+b_{2}$.

8. Consider now the stationary distribution of the $a_{i}$ which is reached in the stationary Gauss process (1), (2). It is

$$
\varrho_{s}\left(\left\{a_{i}\right\}\right) \propto \exp \left(-\frac{1}{2} a_{i} \Gamma_{j i}^{-1} a_{j}\right)
$$

and $\Gamma_{i j}$ determined by Equation (16). The proof given below will show the important role of the anti-relaxation solution of the variational principle. This adds to the observation of Waldmann's that only the relaxation solution survives, if $t_{2} \rightarrow+\infty$ (or only the anti-relaxation solution, if $t_{1} \rightarrow-\infty$ ); for finite time intervals both solutions contribute.

To prove Eq. (20) one has to sum $\varrho\left(\left\{a_{i}(t)\right\}\right)$ over all paths which start at $a\left(t_{1}\right) \rightarrow 0$ for $t_{1} \rightarrow-\infty$ and end up at $a\left(t_{2}\right)=a$ for $t_{2}$, say $t_{2}=0$. This sum is $N \exp \left(-S_{\min }\right), N$ normalization, $S_{\min }$ the action at the minimum. To calculate it take the general solution (14).

Since $a(-\infty)=0$ choose $b_{2}=0$. With $a(0)=a$ and the definition (16) for $\Gamma$ one gets $b_{1}=\Gamma^{-1} a$. Thus

$$
a(t)=\int_{-\infty}^{t} \mathrm{~d} t^{\prime} e^{\lambda\left(t^{\prime}-t\right)} D e^{\tilde{\lambda} t^{\prime}} \Gamma^{-1} a .
$$

Calculate now $S_{\min }$ by inserting the most probable path $(21)$ into the action $\left(4^{\prime}\right)$.

$$
\begin{aligned}
S_{\min } & =\frac{1}{2} \int_{-\infty}^{0} \mathrm{~d} t(\dot{a}(t)+\lambda a(t))^{\sim} D^{-1}(\dot{a}(t)+\lambda a(t)) \\
& =\frac{1}{2} \int_{-\infty}^{0} \mathrm{~d} t\left(D e^{\tilde{\lambda} t} \Gamma^{-1} a\right)^{\sim} D^{-1}\left(D e^{\tilde{\lambda} t} \Gamma^{-1} a\right)
\end{aligned}
$$




$$
\begin{aligned}
& =\frac{1}{2} \tilde{a} \Gamma^{-1} \int_{-\infty}^{0} \mathrm{~d} t e^{\lambda t} D e^{\tilde{\lambda} t} \Gamma^{-1} a \\
& =\frac{1}{2} \tilde{a} \Gamma^{-1} a .
\end{aligned}
$$

This verifies (20). Note that the anti-solution (21) was essential for the proof, while the relaxation term $\propto \exp (-\lambda t)$ has already died out.

9. In general, the physical system considered is defined by $\lambda$ and $g$, i.e. $\lambda$ and $D$. One then finds the stationary correlations $\Gamma$ from Equation (17).

It may happen (and for proper choice of variables indeed does) that

$$
\lambda+\tilde{\lambda}=D \text {. }
$$

Then $\hat{D}(t)$ of $(15)$ simplifies.

$$
\hat{D}(t)=1-e^{-\lambda t} e^{-\tilde{\lambda} t},
$$

and

$$
\Gamma=\lim _{t \rightarrow \infty} \hat{D}(t)=1 .
$$

Conversely, $\Gamma=1$ implies (22) and $L=\lambda$. Then $\lambda D-D \tilde{\lambda}=\lambda^{2}-\tilde{\lambda}^{2}$; necessary and sufficient condition for the property (6) then is $\lambda=\tilde{\lambda}$. In general, $\lambda \neq \tilde{\lambda}$, even if there is Onsager symmetry $\left(5^{\prime}\right)$, $\theta \lambda=\tilde{\lambda} \theta$, as always at thermal equilibrium. So $\left(5^{\prime}\right)$ may hold but not (6), e.g. if even and odd variables are mixed.

10. Some final remarks: i) If the symmetry property (6) holds, the integral in (16) can easily be performed, $\Gamma=\frac{1}{2} \lambda^{-1} D$, i.e. $2 \lambda \Gamma=D$, as already mentioned.

ii) In equilibrium $\Gamma \propto T$, temperature. Equation (17) then says that the fluctuation strength $D$ and the relaxation matrix $\lambda$ are proportional, the factor being the temperature T. Thus Eq. (17) is a general

[1] L. Waldmann, Phys. Letters 63 A, 196 (1977).

[2] L. Onsager and S. Machlup, Phys. Rev. 91, 1505, 1512 (1953).

[3] I. Prigogine and R. Lefever, J. Chem. Phys. 48, 1695 (1968), see also: G. Nicolis, and I. Prigogine, Self-Organization in Non-Equilibrium Systems; Wiley Inscience, New York 1977.

[4] R. Graham, Springer Tracts in Modern Physics, Vol. 66, 1973. form of the (static) fluctuation-dissipation theorem. Another form is Equation (16).

iii) Microscopic time reflection symmetry (more precisely : $L=\widetilde{L}$ ) not only is a sufficient but also a necessary condition for the symmetry (6) of $\lambda D$.

iv) Time reflection symmetry of the process (2) itself means $\theta \lambda \theta=-\lambda, \theta D \theta=D$. Equation (17) then implies $D=-D=0$ and thus $L=\lambda \Gamma=-\tilde{L}$. So reversible mechanics or electrodynamics are limiting cases of the variational principle (4). Note that the latter is independent of the magnitude of $D$. A positive real part in the eigenvalues of $\lambda$ together with $D \neq 0$ necessarily implies irreversibility.

v) The limiting case of a reversible system can be incorporated in the variational principle by adding infinitesimal irreversibility, $\lambda=\lambda_{\text {rev }}+\varepsilon 1$, with $\theta \lambda_{\text {rev }} \theta=-\lambda_{\text {rev }}$. To fulfill the fluctuation-dissipation relation (17), also $D$ has to be infinitesimal $D=2 \varepsilon \Gamma$, and $\lambda_{\mathrm{rev}} \Gamma+\Gamma \tilde{\lambda}_{\mathrm{rev}}=0$. If $\theta \lambda_{\mathrm{rev}}=\tilde{\lambda}_{\mathrm{rev}} \theta$ one can choose $\Gamma=1$. The equation of motion implied by the variational principle is $\left(\partial_{t}+\lambda_{\text {rev }}\right)^{2} a$ $=0$. It is linear, of second order, time reversal invariant term by term.

vi) The stochastically equivalent Fokker-Planck equation for the process (1), (2) is

$$
\partial_{t} \varrho(a, t)=\left(\partial_{i} \lambda_{i j} a_{j}+\frac{1}{2} \partial_{i} \partial_{j} D_{i j}\right) \varrho(a, t) .
$$

vii) $\hat{D}(t)$ defined in (15) has a physikal meaning. It is the momentary correlation matrix

$$
\hat{D}_{i j}(t)=\left\langle\left(a-\langle a\rangle_{t}\right)_{i}\left(a-\langle a\rangle_{t}\right)_{j}\right\rangle_{t} .
$$

Its eqation of motion is

$$
\delta_{t} \hat{D}(t)=D-\lambda \hat{D}(t)-\hat{D}(t) \hat{\lambda},
$$

c. f. Equation (18).

I would like to thank Prof. L. Waldmann for his interest and his comments.

[5] R. F. Fox and G. E. Uhlenbeck, Phys. Fluids 13 1893, 2881 (1970).

[6] L. Waldmann, Z. Naturforsch. 31 a, 1029 (1976).

[7] L. Onsager, Phys. Rev. 37, 405 (1931); 38, 2265 (1931).

[8] S. Grossmann, R. Schranner, and P. H. Richter, Z. Phys. B, 1979, in print.

[9] L. D. Landau and E. M. Lifschitz, Statistical Physics, Addison-Wesley, Reading, MA, 1973, Sec. 122/4.

[10] F. Jähnig and P. H. Richter, J. Chem. Phys. 64, 4645 (1976). 